Von der Forschungsprofessur für Theoretische Physik dex Liniversität Hamburg

\title{
Elektrostatische Felder in der erweiterten Gravitationstheorie
}

\author{
Von \\ JÜRGEN EHLERS \\ (Eingegangen am 20. August 1956)
}

In dieser Arbeit soll eine Methode zur Integration relativistischer Feldgleichungen, die ich in einer früheren Arbeit ${ }^{1}$ auf die EinsteIsschen, die EINSTEIN-MIs XwELLschen und die JORDANschen Gleichungen angewandt habe, auf die JORDAN-MAXwelLschen Feldgleichungen übertragen werden. Die Integration wird dadurch geleistet, daß die Feldvariablen als voneinander funktional abhängig angenommen werden. So könnell einfache Quadraturen zur Bestimmung dieser Abhängigkeiten von den Hauptgleichungen abgespalten und diese zugleich vereinfacht werden. Die gewonnene Lösungsklasse kann als Analogon der zuerst von A. PAPAPETROU ${ }^{2}$ und unabhängig davon von S. D. M.AJUMDAR ${ }^{3}$ gefundenen Lösung in der erweiterten Gravitationstheorie angesehen werden *. Als Beispiel gebe ich das statische kugelsymmetrische elektro-gravische Feld, daß der ReIssner-Xondoströmschen Lösung in der klassischen Relativitätstheorie entspricht, an.

\section{Die ScHÜCKINGsche Transformation für elektromagnetische Felder}

Die Vakuumfeldgleichungen für elektromagnetisch-gravische Felder in der erweiterten Gravitationstheorie lassen sich aus dem Variationsprinzip

$$
\delta \int x\left(R-\zeta \frac{\partial_{j} x \partial^{j} x}{\varkappa^{2}}+\frac{\varkappa}{2} F_{b l} F^{k}\right) \sqrt{-g} d x=0
$$

ableiten ${ }^{4}$. Darin bedeutet $R$ den Krümmungsskalar, die Spur des RIcci-Tensors $R_{j k}$, zu der natürlichen Metrik

$$
Q=g_{k l} d x^{k} d x^{l}
$$

$\varkappa$ die (veränderliche) Gravitationsinvariante, $F_{k l}=2 \partial_{[k} A_{l]}$ den MAXweLLschen Feldstärkentensor, der sich aus dem Viererpotential $A_{l}$ ableitet, und $\zeta$ eine dimensionslose Konstante. $\nabla_{k}$ bezeichnet den Operator der kovarianten Ableitung, $\nabla^{k}=g^{k l} \Gamma_{l}$ den der kontravarianten; $\partial_{j}$ bedeutet gewöhnliche Differentiation nach $x^{j}, \partial^{j}=g^{j l} \partial_{l} . B_{[k l]}=\frac{1}{\underline{z}}\left(B_{k l}-B_{l \bar{k}}\right)$ ist der schiefsymmetrische Anteil des Größensystems $B_{k} t$. Die Signatur

* Die Arbeit PaPAPETRous wurde praktisch gleichzeitig mit der MaJuMdars eingereicht. Die von mir früher betonte Priorität MajumDars besteht relativ zu mir, nicht zu Papapetrou.

2 Ehlers, J.: Z. Physik 143, 239-248 (1955).

2 Papapetrou, A.: Proc. Roy. Ir. Acad. 51, 191 (19+7).

3 Majumdar, S.D.: Phys. Rer. 72, 390 (1947).

4 JoRdAn, P.: Schwerkraft und Weltall, 2. Aufl., S. 164, (7). Braunschweig 1955. 
von (2) wählen wir mit PAULI als $(+++-)$; wir setzen die Lichtgeschwindigkeit und die Einsteinsche Gravitationskonstante $=1$. In (1) sind $\%, g_{k l}$ und $A_{l}$ zu variieren.

Die genannten Feldgleichungen vereinfachen sich wie im Falle eines reinen Gravitationsfeldes erheblich, wenn man nach ScHücKING ${ }^{5}$ von (2) zu der konform abgeänderten Hilfsmetrik

$$
\bar{g}_{k l}=x g_{k l}
$$

übergeht. Alle auf die neue Metrik bezogenen Größen sollen durch einen Querstrich gekennzeichnet werden. Aus (3) folgt nach bekannten Formeln 6

und

$$
R=\varkappa\left(\bar{R}+3 \varkappa \bar{\nabla}_{k}^{k}\left(\frac{1}{x}\right)-\frac{3}{2} \frac{\partial_{k} \varkappa \bar{\partial}^{k} x}{\varkappa^{2}}\right)
$$

$\varkappa \sqrt{-g}=\frac{\sqrt{-\bar{g}}}{\varkappa}, \quad \frac{\partial_{j} \varkappa \partial^{j} \varkappa}{\varkappa^{2}}=\frac{\partial_{j} \varkappa \bar{\partial}^{j} \varkappa}{\varkappa}, \quad F_{k l} F^{k l}=\varkappa^{2} F_{k l} \bar{F}^{k l}$.

(1) ist also äquivalent mit

$\delta \int\left(\bar{R}+3 \varkappa \bar{\nabla}_{k}^{k}\left(\frac{1}{\varkappa}\right)-\left(\zeta+\frac{3}{2}\right) \frac{\partial_{j} \varkappa \bar{\partial}^{j} \varkappa}{\varkappa^{2}}+\frac{\varkappa^{2}}{2} F_{k l} \bar{F}^{k} l\right) \sqrt{-\bar{g}} d x=0$.

Wenn wir $\varkappa=e^{u}$ setzen, ist

$$
3 \varkappa \bar{\nabla}_{k}^{k}\left(\frac{1}{\varkappa}\right)=3\left(\partial_{j} u \bar{\partial}^{j} u-\bar{\nabla}_{j}^{j} u\right), \quad \frac{\partial_{j} x}{\varkappa}=\partial_{j} u .
$$

Das Variationsprinzip

$\delta \int\left(-\frac{1}{2} \bar{R}+\alpha \partial_{k} u \bar{\partial}^{k} u-\frac{1}{4} e^{2 u} F_{k l} \bar{F}^{k}\right) \sqrt{-\bar{g}} d x=0 \quad\left(\alpha=\frac{1}{2}\left(\zeta-\frac{3}{2}\right)\right)(7)$ ist also gleichwertig mit (1). Wir setzen $\alpha \neq 0$ voraus.

(7) kann formal als Hamiltonsches Prinzip für ein Feld in der klassischen EINSTEINschen Theorie gedeutet werden; als Feldvariablen sind der Skalar $u$ und der Vektor $A_{l}$ anzusehen; die LAGRANGE-Dichte ist

$$
\bar{\Omega}=\alpha \bar{g}^{k l} \partial_{k} u \partial_{l} u-\frac{1}{4} e^{2 u} \bar{g}^{k v} \bar{g}^{l s} F_{k l} F_{r s} .
$$

Danach haben wir die Feldgleichungen

und

$$
-\bar{R}_{k l}=\bar{T}_{k l}-\frac{1}{2} \bar{g}_{k l} \bar{T}
$$

mit

$$
\bar{\Omega}_{u}=\partial_{k}\left(\bar{\Omega}_{\hat{\delta}_{k} u}\right), \quad \partial_{k}\left(\bar{\Omega}_{\partial_{k} A_{l}}\right)=0
$$

$$
\frac{1}{2} \overline{\mathfrak{I}}^{k l}=\frac{\hat{\partial} \overline{\mathbf{I}}}{\partial \vec{g}_{k l}} .
$$

\footnotetext{
5 JORDAN, P.: a. a. O. $\$ 32$.

6 Jordan, P.: a. a. O. \$11, (45).
} 
Die Definition (11) des Energie-Impuls-Tensors ist, falls $\bar{\Omega}=\bar{L} \sqrt{-\bar{g}}$, $\bar{L}=\bar{L}\left(\psi, \partial_{k} \psi, \ldots, \bar{g}^{k l}\right)-\psi$ steht als Abkürzung für die Feldvariablen ist, mit der einfacheren Formel

gleichwertig*. Setzen wir

$$
\bar{T}_{k l}=\bar{L} \bar{g}_{b l}-2 \bar{L}_{\bar{g}^{k l}}
$$

$$
\bar{E}_{k l}=\bar{F}_{k m} \bar{F}_{l}^{\prime \prime n}-\frac{1}{4} \bar{g}_{k l} \bar{F}_{r s} \bar{F}^{r s}, \quad\left(\bar{F}_{k l}=F_{k l}\right)
$$

so liefert (12)

$$
\bar{T}_{k l}=-2 \alpha \partial_{k} u \partial_{l} u+\alpha \partial_{m} u \bar{\partial}^{m} u \bar{g}_{k l}+e^{2 u} \bar{E}_{k l} .
$$

Die Feldgleichungen (9) sehen also so aus:

$$
-\bar{R}_{k l}=-2 \alpha \partial_{k} u \partial_{l} u+e^{2 u} \bar{E}_{k l} .
$$

Die LAGRANGE-Gleichungen (10) nehmen die Form

$$
\bar{\nabla}_{l}\left(e^{2 u} \bar{F}^{k l}\right)=0, \quad \alpha \bar{\nabla}_{k}^{k} u+\frac{1}{4} e^{2 u} \bar{F}_{k l} \bar{F}^{k l}=0
$$

an. Wenn man statt $A_{l} F_{k l}$ als Feldgröße benutzen will, muß man zu (15) und (16), dem Gravitationsgesetz und der einen MaxwelLschen Gleichung, noch die andere Maxwell-Gleichung

hinzunehmen.

$$
\partial_{[i} F_{k l]}=0
$$

Die zweite Gl. (16) ist im wesentlichen eine Folge der Gl. (15), der ersten Gl. (16) und (17). Aus (15), (17) und der ersten Gl. (16) folgt nämlich

$$
0=\bar{\nabla}_{k} \bar{T}_{l}^{k}=-2 \partial_{l} u\left(\alpha \cdot \bar{V}_{k}^{k} u+\frac{1}{4} e^{2 u} \bar{F}_{r s} \bar{F}^{r s}\right) .
$$

Daraus entnehmen wir folgenden Satz: Jede Lösung der Gln. (15), (17) und der ersten Gl. (16) gehört genau einer der folgenden Klassen an:

1. Es ist $u=$ const und $\bar{F}_{k l} \bar{F}^{k l}=0$. Dann löst $\left(\bar{g}_{k l}, \bar{F}_{k l}, u\right)$ die transformierten JORDAN-MAXWELLschen Feldgleichungen, und zugleich befriedigt $\left(\bar{g}_{k l}, \widetilde{F}_{k l}\right)$ mit $\widetilde{F}_{k l}=e^{u} \bar{F}_{k l}$ die Enstein-Maxwelischen Feldgleichungen.

2. Es ist $u=$ const und $\bar{F}_{k l} \bar{F}^{k} l \neq 0$. Dann löst $\left(\bar{g}_{k l}, \bar{F}_{k l}, u\right)$ die JORDANMAXwELLschen Gleichungen nicht, wohl aber befriedigt $\left(\bar{g}_{k l}, \widetilde{F}_{k l}\right)$ die Einstein-Maxwellschen Feldgleichungen.

* Soweit ich weiß, tritt die einfache und allgemein gültige Formel (12), die man leicht aus (11) ableiten kann und die man auch in der speziellen Relativitätstheorie verwenden kann, in der Literatur nicht auf. $\boldsymbol{T}_{k l}$ kann so ohne den Umweg über den ,kanonischen Tensor " und anschließende Symmetrisierung (BeLINFanTE) bestimmt werden. Aus (12) folgt, daß die Spur des Energietensors genau dann verschwindet, wenn $L$ in der $g^{k l}$ homogen vom Grade 2 ist wie beim MAXwelLFeld. Die drei Eigenschaften ", $T=0 ",{ }^{\prime} L$ ist honogen vom Grade 2 in den $g^{k l "}$ und " $L$ ist konforminvariant" sind also gleichwertig. 
3. Es ist $u \neq$ const. Dann löst $\left(\bar{g}_{k l}, \bar{F}_{k}, \imath\right)$ die Jordan-MaXWeLLschen Feldgleichungen.

Durch diesen Satz wird erstens das Verhältnis der Lösungen der Jordan-Maxwelzschen Theorie zu denẹn der Einstein-MaXwelischen Theorie festgestellt, und zweitens ergibt sich, daß das uiber die klassische Theorie hinausführende mathematische Problem der erweiterten Gravitationstheorie in der Lösung der Gln. (15), (17) und der ersten Gl. (16) besteht. Durch die ScHückingsche Transformation wird also die Anzahl der Feldgleichungen wie im Falle reiner Gravitationsfelder um eins vermindert.

\section{Elektrostatische Felder}

Elektrostatische Felder definieren wir durch die Forderungen, daß für die natürliche Metrik (2) und das Viererpotential $A_{l}$ gelten soll:

$$
\partial_{0} g_{k l}=0, \quad g_{0 v}=0, \quad \partial_{0} \varkappa=0, \quad \partial_{0} A_{0}=0, \quad A_{v}=0 .
$$

$x^{0}=t$ soll die Zeitkoordinate, $x^{v}$ sollen die Raumkoordinaten bedeuten. Griechische Indizes laufen von 1 bis 3, lateinische von 0 bis 3 .

Wegen (3) sind diese Bedingungen, soweit sie $g_{k l}$ betreffen, mit den analogen Beziehungen für $\bar{g}_{k l}$ gleichwertig. Für die durch $\vec{g}_{k l}$ bestimmte metrische Fundamentalform $\bar{Q}$ können wir also den bei statischen Feldern bewährten Ansatz

$$
\bar{Q}=e^{-2 \Omega} d l^{2}-e^{2 \Omega} d t^{2}, \quad d l^{2}=\gamma_{\mu \nu} d x^{\mu} d x^{\nu}
$$

machen. $d l^{2}$ ist eine positiv definite Metrik des dreidimensionalen Raumes, $\Omega=\Omega\left(x^{\nu}\right)$ ein räumlicher Skalar.

Wir wollen die Feldgleichungen (15) und (16) in Tensorgleichungen, die sich auf den Rautm mit der Metrik $a l^{2}$ beziehen, umschreiben. Dazu stützen wir uns auf den

Hilfssatz: Ist $\bar{Q}=e^{-2 \Omega} d l^{2}-e^{2 \Omega} d t^{2}$ die metrische Fundamentalform eines vierdimensionalen Raumes, $\Omega$ ein Skalar in dem dreidimensionalen Raum mit der Metrik $d l^{2}$, so bestehen zwischen den RiccI-Tensoren $\bar{R}_{k l}$ und $P_{r \lambda}$ die Beziehungen

$\bar{R}_{00}=-e^{4 \Omega} \nabla_{\nu}^{\nu} \Omega ; \quad \bar{R}_{0 \nu}=0, \quad \bar{R}_{\mu \nu}=P_{\mu \nu}-\gamma_{\mu \nu} \nabla_{\lambda}^{\lambda} \Omega+2 \partial_{\mu} \Omega \partial_{\nu} \Omega$.

Der Beweis soll hier nicht ausgeführt werden; er ergibt sich etwa aus den Formeln (2) und (14) meiner Arbeit „Exakte Lösungen ..." 7 . Aus (9) werden dann mit (14) die Gleichungen

$$
\begin{gathered}
e^{2 \Omega} \nabla_{\nu}^{\nu} \Omega=\frac{1}{2} e^{2 u} \partial_{\nu} \Phi \partial^{\nu} \Phi, \\
P_{\mu \nu}=-2 \partial_{\mu} \Omega \partial_{\nu} \Omega+2 \alpha \partial_{\mu} u \partial_{\nu} u+e^{2(u-\Omega)} \partial_{\mu} \Phi \partial_{\nu} \Phi,
\end{gathered}
$$

7 Ehlers, J.: Z. Physik 140, 394-408 (1955). 
und die erste der Beziehungen (16) geht, wenn $-A_{0}=\Phi$ (elektrostatisches Potential) gesetzt wird, über in

$$
\nabla_{p}\left(e^{2(u-\varrho)} \hat{d}^{p} \Phi\right)=0 .
$$

(21), (22) und (23) bilden das vollständige System der elektrostatischen Feldgleichungen, bezogen auf den Raum mit der Metrik $d l^{2}$, die durch

$$
Q=e^{-n-2 \Omega} d l^{2}-e^{-u+2 \Omega} d t^{2}
$$

mit der Weltmetrik verknüpft ist.

Aus den Feldgleichungen (21), (22) und (23) kann man mit Hilfe eines Satzes von E. HopF über elliptische Differentialgleichungen auf Mannigfaltigkeiten ${ }^{8}$ schließen:

Satz: Die einzige singularitätenfreie statische Lösung der JoRDANMaxwellschen Feldgleichungen, bei der der Raum kompakt ist oder einen einzigen unendlich fernen Punkt (wie der euklidische Raum im Sinne der Potentialtheorie) hat, in $\operatorname{dem} \varkappa, \Phi$ und $\Omega$ endlich sind, ist die Minkowski-Welt mit $x=$ const und $\Phi=$ const.

Beweis: Nach (23) genügt $\Phi$ einer elliptischen Differentialgleichung, ist also nach $\mathrm{E}$. Hopf konstant. Daraufhin muß aus demselben Grund wegen (21) $\Omega=$ const und wegen (22) (ScHÜCKINGscher Satz) $u=$ const sein. Wegen $P_{u \nu}=0 \mathrm{mu} B d l^{2}$ euklidisch, wegen $u=$ const und $\Omega=$ const also $Q$ Minkowskisch sein.

Dafür, daß der entsprechende Satz für Räume mit zwei unendlich fernen Punkten nicht mehr gilt, werden wir unten ein Beispiel angeben.

Der Erhaltungssatz für den Ricci-Tensor $P_{\mu}$, liefert für jede Löstung von (22) die Gleichung

$$
\left.\begin{array}{rl}
0=\nabla_{\mu}\left(e^{2(u-\Omega)} \partial^{\mu} \Phi\right) \cdot \partial_{\nu} \Phi & +\left(e^{2(u-\Omega)} \partial_{\lambda} \Phi \partial^{\lambda} \Phi-2 \nabla_{u}^{\mu} \Omega\right) \cdot \partial_{\nu} \Omega+ \\
& +\left(2 \alpha \nabla_{\mu}^{\mu} u-e^{2(u-\Omega)} \partial_{\lambda} \Phi \partial^{\lambda} \Phi\right) \partial_{\nu} u .
\end{array}\right\}
$$

Daraus ist abzulesen: Wenn (21), (22) und (23) erfüllt sind und $u$ nicht konstant ist, gilt auch

$$
\nabla_{\nu}^{v} u=\frac{1}{2 \alpha} e^{2(u-2)} \partial_{\lambda} \Phi \hat{\sigma}^{i} \Phi .
$$

Außerdem folgt aus (25) der

Satz: Wenn die drei Skalare $\Omega, u, \Phi$ unabhängige Funktionen sind, so folgen (21) und (23) aus (22), d.h. diese elektrostatisch-gravischen

$8 \mathrm{Vgl}$. Lichnerowicz, A., Theories relativistes de la gravitation et de l'electromagnetisme, Paris 1955 , p. 130. Der dort angegebene Satz kann leicht verallgemeinert werden zu folgendem: Ist $V_{n}$ eine zweimal stetig differenzierbare Mannigfaltigkeit, $L=a^{\mu \nu} \hat{c}_{\mu \nu}+b^{\nu} \hat{c}_{\nu}$ ein auf $V_{n}^{r}$ stetiger homogener elliptischer Differentialoperator zweiter Ordnung und genügt die auf $V_{n}$ zweimal stetig differenzierbare Funktion $U^{r}$ der Ungleichung $L\left(C^{T}\right) \geq 0$, so ist $U=$ const auf $V_{n}$, wenn $V_{n}^{\prime}$ entweder lompakt ist oder einen unendlich fernen Punlet hat, in dem $U$ einen. endlichen Grenzwert annimmt. Lichnerowtcz beweist ähnliche Sätze wie den oben angegebenen für andere Feldgleichungen. 
Felder der erweiterten Gravitationstheorie sind vollständig durch die Gl. (22) bestimmt.

Wir wollen uns im folgenden mit den Lösungen von (21), (22) und (23) beschäftigen, bei denen $w$ und $\Phi$ nicht konstant und je zwei der drei Funktionen $\Omega, u, \Phi$ voneinander abhängig sind, wie es z.B. bei kugelsymmetrischen, zylindersymmetrischen und ebenen Feldern der Fall ist. Für eine solche Lösung können wir

$$
e^{2(\Omega-u)}=g(\Phi)
$$

setzen. Dann folgt durch Differentiation von (27)

$$
d \Omega-d u=\frac{1}{2} \frac{g^{\prime}}{g} d \Phi
$$

und durch nochmalige Differentiation und Einsetzen von (21), (23) und (26) für $g$ die elementare Differentialgleichung $g^{\prime \prime}=1-\frac{1}{\alpha}$, also muß

$$
e^{2(\Omega-u)}=\beta \Phi^{2}+A \Phi+B \quad\left(\beta \equiv \frac{1}{2}\left(1-\frac{1}{\alpha}\right)\right)
$$

sein. Führen wir die Hilfsfunktion $V$ durch

$$
d V=e^{2(u-\Omega)} d \Phi=\frac{d \Phi}{g(\Phi)}
$$

ein, so geht (23) über in die homogene Potentialgleichung

$$
\nabla_{v}^{v} V=0 \text {. }
$$

Aus $\nabla_{\nu}\left(\frac{\partial^{y} \Phi}{g}\right)=0$ ergibt sich die Hilfsformel

$$
\nabla_{v}^{\nu} \Phi=\frac{g^{\prime}}{g} \partial_{\nu} \Phi \partial^{\nu} \Phi
$$

Wegen der oben ausgesprochenen Voraussetzungen besteht außerdem eine Relation

$$
\Omega=h(\Phi)
$$

aus der $\nabla_{v}^{v} \Omega=h^{\prime} \nabla_{v}^{v} \Phi+h^{\prime \prime} \partial_{\nu} \Phi \partial^{n} \Phi$ folgt. In Verbindung mit (22) und (32) ergibt sich daraus $\left(h^{\prime} g\right)^{\prime}=\frac{1}{2}$, also ist $h^{\prime} g=\frac{1}{2} \Phi+C$. Da eine Änderung von $\Phi$ um eine additive Konstante weder die Weltmetrik noch das elektromagnetische Feld und die Gravitationsinvariante beeinflußt, können wir ohne Beschränkung der Allgemeinheit $C=\frac{1}{2} A$ setzen:

$$
h^{\prime} g=\frac{1}{2}(\Phi+A) .
$$

Dafür können wir wegen (33) und (30)

$$
d \Omega=\frac{1}{2}(\Phi+A) d V
$$


schreiben. Daraus und aus (28), (29) und (30) folgt

$$
d u=\left(\frac{1}{2}-\beta\right) \Phi d V
$$

Wenn wir (35), (36), (30) und (27) in (22) einsetzen, erhalten wir

$$
\begin{aligned}
& \frac{1}{2} P_{\mu \nu} d x^{n l} d x^{n}=-(d \Omega)^{2}+\alpha(d u)^{2}+\frac{1}{2 g}(d \Phi)^{2} \\
& \quad=\left(-\left(\frac{1}{2} \Phi+\frac{A}{2}\right)^{2}+\frac{1}{4 \alpha} \Phi^{2}+\frac{1}{2}\left(\frac{1}{2}\left(1-\frac{1}{\alpha}\right) \Phi^{2}+A \Phi+B\right)\right)(d V)^{2} \\
& \quad=\left(\frac{B}{2}-\frac{A^{2}}{4}\right)(d V)^{2} .
\end{aligned}
$$

Es gilt also mit $a=\frac{A^{2}}{4}-\frac{B}{2}$ :

$$
P_{\mu \nu}+2 a \hat{c}_{\mu} \mathrm{F}^{-} \hat{c}_{2} \mathrm{~V}=0 .
$$

Als Zusammenfassung dieser Überlegungen formulieren wir folgenden

Satz: Diejenigen Lösungen der Gln. (21), (22) und (23), bei denen $u$ und $\Phi$ nicht konstant und je zwei der drei Skalare $Q, u, \Phi$ voneinander abhängig sind, sind vollständig bestimmt durch die Relationen

$$
\begin{gathered}
P_{\mu \nu}+2 a \delta_{\mu} V \dot{c}_{\nu} V=0, \\
g(\Phi)=\beta \Phi^{2}+A \Phi+\frac{A^{2}}{2}-2 a, \\
\frac{d \Phi}{g}=d V, \\
d \log \%=\left(\frac{1}{2}-\beta\right) \Phi d V .
\end{gathered}
$$

Darin ist

$$
\beta=\frac{1}{2}\left(1-\frac{1}{\alpha}\right)=\frac{2 \zeta-7}{4 \xi-6}+\frac{1}{2} \text {. }
$$

Außerdem muß

$$
g(\Phi)>0 \text { und } \varkappa>0
$$

und für $a=0$

$$
\Delta V=0
$$

sein. Die Weltmetrik hat die Gestalt

$$
Q=\left(\varkappa^{3} g(\Phi)\right)^{-1} d l^{2}-\varkappa g(\Phi) d t^{2} .
$$

Wir haben eben schon bewiesen, daß die im Satz angegebenen Gleichungen notwendige Bedingungen für Lösungen dieser Art sind. Sie sind auch hinreichend, wie eine einfache Rechnung zeigt. Dabei ist der ScHÜckINGsche Satz zu berücksichtigen, der aussagt, daß aus (37) für $a \neq 0$ die homogene Potentialgleichung für $V^{r}$ folgt (vgl. Fußnote 5). Das Wesentliche an dem Satz ist die Aussage, daP man aus jeder Lösung der Gl. (37) durch zwei Quadraturen eine Lösung der 
statischen JORDAN-MAXweLLschen Gleichungen erhält. Dieses Ergebnis ergänzt einen in meiner früheren Arbeit ${ }^{9}$ abgeleiteten Satz. Verbindet man die Aussagen jener Arbeit mit unserem jetzigen Ergebnis, so ergibt sich die bemerkenswerte Tatsache, daß aus jeder statischen Lösung der ErssteInschen Vakuum-Gravitationsgleichungen durch zwei Quadraturen eine Lösung der JORDAN-MAXWELLschen Gleichungen gewonnen werden kann. Außerdem können die Papapetrou-Majumdarschen (s. oben) und die WeyLschen ${ }^{10}$ Lösungen in die erweiterte Gravitationstheorie übertragen werden. Erstere erhält man für $a=0$ ( $d l^{2}$ euklidisch), letztere, wenn man $d l^{2}$ axialsymmetrisch wählt.

Die Quadraturen (39) und (40) können wegen (38) elementar ausgeführt werden unabhängig von der Lösung der Hauptgleichung (37). Dabei darf über eine additive Konstante in $V$ frei verfügt werden. Es ergeben sich folgende Fälle:

1. $\beta=A=0, \alpha<0$.

$$
g(\Phi)=-2 a, \quad \Phi=-2 a V, \quad \varkappa=B e^{-\frac{a}{2} V^{2}}, \quad B>0 .
$$

2. $\beta=0, \quad A \neq 0$.

$$
\begin{gathered}
g(\Phi)=A \Phi+\frac{A^{2}}{2}-2 a=e^{A V}, \quad \Phi=\frac{1}{A}\left(e^{A V}+2 a-\frac{A^{2}}{2}\right), \\
\varkappa=B \exp \left(\frac{1}{2 A^{2}} e^{A V}+\left(\frac{a}{A^{2}}-\frac{1}{4}\right) A V\right), \quad B>0 .
\end{gathered}
$$

In den Fällen mit $\beta \neq 0$ ist es zweckmäßig, die Substitutionen

vorzunehmen. Dann wird

$$
\Phi \rightarrow \Phi-\frac{A}{2 \beta}, \quad \frac{A^{2}}{2 \beta}-\frac{A^{2}}{4 \beta^{2}}-\frac{2 a}{\beta} \rightarrow A
$$

und

$$
g(\Phi)=\beta\left(\Phi^{2}+A\right)
$$

was sich wegen (39) zu

$$
d \log \chi=\left(\frac{1}{2}-\beta\right)\left(\Phi \pm \sqrt{\frac{\beta A+2 a}{\beta(2 \beta-1)}}\right) d V,
$$

$$
\varkappa=B(g(\Phi))^{\frac{1}{1 \beta}-\frac{1}{2}} \exp \left( \pm \frac{1}{2} \sqrt{(2 \beta-1)\left(A+\frac{2 a}{\beta}\right)} V\right), \quad B>0
$$

integrieren läßt. Es gibt dann noch folgende Fälle:

$$
\begin{aligned}
3 . \beta>0, A & =0 \\
g(\Phi)=\beta \Phi^{2}=\frac{1}{\beta V^{2}}, & \Phi=-\frac{1}{\beta} V \\
\varkappa & =B \cdot|V|^{1-\frac{1}{2 \beta}} \exp \left( \pm \sqrt{\left(1-\frac{1}{2 \beta}\right) a}\right) .
\end{aligned}
$$

Dabei muß $B>0,\left(1-\frac{1}{2 \beta}\right) a \geq 0$ und $V \neq 0$ sein.

9 Ehlers, J.: Z. Physik 143, 239-248 (1955).

10 Weyl, H.: Ann. Phys. 54, 117 (191/); 59 (1919). 
t. $\beta>0, \quad A \equiv\left(\frac{b}{\beta}\right)^{2}>0$.

$g(\Phi)=\beta\left(\Phi^{2}+\left(\begin{array}{c}b \\ \beta\end{array}\right)^{2}\right)=\frac{b^{2}}{\beta \cos ^{2}\left(b I^{2}\right)}, \quad \Phi=\frac{b}{\beta} \operatorname{tg}(b V), \quad b F+(2 n+1) \frac{\pi}{2}$,

$\varkappa=B|\cos b V|^{1-\frac{1}{2 \beta}} \exp \left\{ \pm \frac{1}{2 \beta} \sqrt{(2 \beta-1)\left(b^{2}+2 a \beta\right)} \mathrm{V}\right\}$.

$$
B>0, \quad(2 \beta-1)\left(b^{2}+2 a \beta\right) \geq 0 .
$$

5. $\beta>0, \quad A=-\left(\frac{b}{\beta}\right)^{2}<0$.

$s(\Phi)=\beta\left(\Phi^{2}-\left(\frac{b}{\beta}\right)^{2}\right)=\frac{b^{2}}{\beta \operatorname{\Xi in}^{2}(b V)}, \quad \Phi=-\frac{b}{\beta} \operatorname{\varpi otg}(b \Gamma), \quad V \neq 0$,

$\varkappa=B \mid \operatorname{Sin} b V^{1-\frac{1}{2 \beta}} \exp \left\{ \pm-\frac{1}{2 \beta} \sqrt{(2 \beta-1)\left(2 a \beta-b^{2}\right)} V\right\}$,

$$
B>0, \quad(2 \beta-1)\left(2 a \beta-b^{2}\right) \geq 0 .
$$

6. $\beta<0, A=-\left(\frac{b}{\beta}\right)^{2}<0$.

$$
\begin{gathered}
g(\Phi)=-\beta\left(\left(\frac{b}{\beta}\right)^{2}-\Phi^{2}\right)=\frac{b^{2}}{-\beta\left(\mathfrak{C D}^{2}(b V)\right.}, \Phi=-\frac{b}{\beta} \operatorname{II}(b T), \\
x=B|\operatorname{Coj} b V|^{1-\frac{1}{2 \beta}} \exp \left\{ \pm \frac{1}{2 \beta} \mid \overline{(1-2 \beta)\left(b^{2}-2 a \beta\right)} V\right\}, \\
B>0, \quad b^{2} \geqq 2 a \beta .
\end{gathered}
$$

\section{Kugelsymmetrische Felder}

Wit wollen als Anwendung des letzten Satzes die kugelsymmetrischen statischen elektro-gravischen Felder der erweiterten Gravitationstheorie bestimmen, also das Analogon der REISSNER-Nordströmschen Lösung.

Wie ich früher (s. Fußnote 9) schon angegeben habe, sehen die kugelsymmetrischen Lösungen der differentialgeometrischen Gl. (37) folgendermaßen aus:

$$
\left.\begin{array}{rl}
a=0: \quad d l^{2} & =d r^{2}+r^{2}\left(d \vartheta^{2}+\sin ^{2} \theta d q^{2}\right), \\
r & ={ }_{r}^{C}+D, \quad 0<r<\infty .
\end{array}\right\}
$$

$$
\left.\begin{array}{rl}
a>0: \quad d l^{2} & =d r^{2}+\left(r^{2}-m^{2}\right)\left(d \vartheta^{2}+\sin ^{2} \vartheta d \varphi^{2}\right), \\
V & =\frac{1}{2 \sqrt{a}} \log C\left(\frac{r+m}{r-m}\right), m \neq 0, \quad m \mid<r<\infty, C>0,
\end{array}\right\}
$$


(47c) ist von Interesse im Hinblick auf den Satz von E. HopF. Definiert man nämlich eine relle analytische Vannigfaltigkeit $W$ als topologisches Produkt der $(\vartheta, \varphi)$-Kugel $(0 \leqq \vartheta \leqq \pi, \varphi \bmod 2 \pi)$ und der $r$-Geraden $(-\infty<r<\infty)$, so wird auf $W$ durch (47c) eine überall reguläre, positiv definite analytische Metrik $d l^{2}$ erklärt, und $V$ ist eine auf $W$ überall reguläre Lösung der Potentialgleichung $\Delta V=0$ bezïglich der Metrik $d l^{2}$ (W ist in bezug auf $d l^{2}$ ein rollständiger Riemansscher Raum im Sinne von $\mathrm{H}$. Hopf und $W$. Rinow). In den beiden unendlich fernen Punkten $(r \rightarrow \infty, r \rightarrow-\infty)$ ron $W$ hat $V$ endliche Werte; trotzdem ist $V$ nicht konstant. Man kann die durch $(47 \mathrm{c})$ gegebene Geometrie dadurch veranschaulichen, daß man die ,Ebene“* $\vartheta=\frac{\pi}{2}$ längentreu auf eine Rotationsfläche im euklidischen $R^{3}$ abbildet; es ergibt sich dabei die Rotationsfläche, die aus der Kurve $\frac{y^{\prime}}{|m|}=$ eoj $\left(\frac{x}{|m|}\right)$ durch Drehung um
die $x$-Achse entsteht.

Daß die in (47b) für $r \rightarrow|m|$ auftretende Singularität $\left(g_{\vartheta \vartheta}=g_{\varphi \varphi} \rightarrow 0\right)$ eine echte Singularität ist (nicht wegtransformierbar), folgt daraus, daß - wie man leicht nachrechnet -- für $r \rightarrow|m|$ das invariante Gausssche Krümmungsmaß der Ebene $\vartheta=\frac{\pi}{2}$ über alle Grenzen wächst. [Der durch ( $47 \mathrm{~b}$ ) definierte Raum ist nicht vollständig].

Natürlich kann man $(47 \mathrm{~b}),(47 \mathrm{c})$ konform auf Gebiete des euklidischen $R^{3}$ abbilden; die entsprechenden Formeln sind:

mit

$$
\left.\begin{array}{rl}
d l^{2} & =d r^{2}+\left(r^{2} \pm m^{2}\right)\left(d \vartheta^{2}+\sin ^{2} \vartheta d \varphi^{2}\right) \\
& =\left(1 \pm \frac{m^{2}}{4 \varrho^{2}}\right)^{2}\left(d \varrho^{2}+\varrho^{2}\left(d \vartheta^{2}+\sin ^{2} \vartheta d \varphi^{2}\right)\right)
\end{array}\right\}
$$

$$
r=\varrho \mp \frac{m^{2}}{4 \varrho} \text {. }
$$

Im Falle (47b) wird das Äußere der Kugel $r=\frac{|m|}{2}$ des $R^{3}$, im Falle ( $\left.47 \mathrm{c}\right)$ der ,punktierte" $R^{3}$, d.h. der $R^{3}$ ohne den Nullpunkt $\varrho=0$, winkeltreu auf unsere Riemansschen Räume abgebildet. Die Räume $(4 ; \mathrm{b}, \mathrm{c})$ sind also beide topologisch verschieden rom euklidischen Raum, aber zueinander homöomorph.

Durch die Formeln (47), (44) und die in Abschn. II unter 1.-6. angegebenen Beziehungen werden die statischen kugelsymmetrischen elektro-gravischen Felder der erweiterten Gravitationstheorie explizit beschrieben. Wegen $a \geqq 0$ zerfällt jeder der sechs Typen (außer 1.) in drei Unterfälle, so daß man 16 Lösungstypen bekommt.

* Eine Fläche $F$ in eimem Riemannschen Raum $R$ heißt ,Ebene“, wenn jede in $F$ gezogene Geodätische zugleich eine Geodätische in $R$ ist. 
Wir wollen hier nicht alle diese Lösungen diskutieren, sondern als Beispiele nur die Fälle mit $\beta=0\left(\zeta=\frac{7}{2}\right)$, also 1 . und 2., behandeln. Dabei machen wir davon Gebrauch, daß wir in den Endformeln die Integrationskonstanten umbenennen und $\Phi$ um eine additive Konstante abändern dürfen.

1. In diesem Fall kann man die Lösung auf die Form bringen:

$$
\begin{gathered}
Q=\frac{d r^{2}+\left(r^{2}+m^{2}\right)\left(d \vartheta^{2}+\sin ^{2} \vartheta d q^{2}\right)}{2 a^{2} B^{3} \exp \left(\frac{3}{2}\left(\operatorname{arctg} \frac{r}{m}+C\right)^{2}\right)}-2 a^{2} B \exp \left(\frac{1}{2}\left(\operatorname{arctg} \frac{r}{m}+C\right)^{2}\right) d t^{2}, \\
\Phi=2 a \operatorname{arctg} \frac{r}{m}, \quad x=B \exp \frac{1}{2}\left(\operatorname{arctg} \frac{r}{m}+C\right)^{2}
\end{gathered}
$$

mit

$$
a>0, \quad m \neq 0, \quad B>0, \quad-\infty<r<\infty .
$$

Die Metrik $Q$ ist zweiseitig $(r \rightarrow \pm \infty)$ asymptotisch Minkowskisch; $\Phi$ und $*$ sind auf der ganzen Mannigfaltigkeit beschränkt. Betrachtet man den Flächeninhalt der Kugeln $r=$ const in Abhängigkeit von $r$, so erkennt man: Für hinreichend große $|C|$ existiert genau eine Kugel kleinsten Flächeninhalts, während nach beiden Seiten die Flächeninhalte monoton wachsen. Für kleine $|C|$ gelangt man bei abnehmendem $|r|$ sowohl von $r=\infty$ als auch von $r=-\infty$ aus zu einer kleinsten Kugel, nach deren Durchschreiten der Flächeninhalt wieder bis zu einem relativen Maximum wächst; in dieser relativ größten Kugel hängen beide Seiten miteinander zusammen.

Für $C=0$ ist die Transformation $r \rightarrow-r$ eine Bewegung, bei der $\Phi$ und $z$ ungeändert bleiben; die beiden Seiten $(r \gg 0, r \ll 0)$ sind synchronisiert. Für $C \neq 0$ herrscht keine solche Symmetrie, und die zwei Seiten des Raumes sind nicht synchronisiert.

Die jetzt betrachtete Lösung belegt unsere auf S. 519 ausgesprochene Behauptung, daß es in der erweiterten Gravitationstheorie statische elektro-gravische Felder gibt, die nicht von Materie erzeugt werden und bei denen der Raum zwei unendlich ferne Punkte hat.

2. $a=0$. Die Lösung sieht dann so aus:

$$
\begin{aligned}
& Q=\frac{d r^{2}+r^{2}\left(d \vartheta^{2}+\sin ^{2} \vartheta d \phi^{2}\right)}{B^{3} \exp \left(\frac{3}{2 A^{2}} \exp \left(\frac{C}{r}+D\right)+\frac{1}{4}\left(\frac{C}{r}+D\right)\right)} \\
& \quad-B \exp \left(\frac{1}{2 A^{2}} \exp \left(\frac{C}{r}+D\right)+\frac{3}{4}\left(\frac{C}{r}+D\right)\right) d t^{2}, \\
& \Phi=\frac{1}{A} \exp \left(\frac{C}{r}+D\right), \quad \%=B \exp \left(\frac{1}{2 A^{2}} \exp \left(\frac{C}{r}+D\right)-\frac{1}{4}\left(\frac{C}{r}+D\right)\right)
\end{aligned}
$$

mit

$$
A \neq 0, \quad C \neq 0, \quad B>0, \quad 0<r<\infty .
$$


Für $r \rightarrow \infty$ ist diese Welt wieder asymptotisch Minkowskisch. Für $r \rightarrow 0$ ist die Lösung singulär. Wenn $C>0$ ist, hat man für $r \rightarrow 0$ totale Violettverschiebung, $|\Phi| \rightarrow \infty, x \rightarrow \infty$, und die Flächeninhalte der Kugeln $r=$ const streben gegen Null. Ist $C<0$, so hat man totale Rotverschiebung, $\Phi \rightarrow 0, x \rightarrow \infty$, der Flächeninhalt der Kugel $r=$ const wächst für $r \rightarrow 0$ über alle Grenzen, also muß eine Minimalkugel existieren ähnlich wie in Fall 1. Im Gegensatz zu 1. wird aber auch für $C<0$ der Raum für $r \rightarrow 0$ nicht asymptotisch Minkowskisch, sondern er wird immer extremer nichteuklidisch.

2. $a>0$. Jetzt ist

$$
\begin{gathered}
Q=\frac{d r^{2}+\left(r^{2}-m^{2}\right)\left(d \vartheta^{2}+\sin ^{2} \vartheta d \varphi^{2}\right)}{8 a A^{2} C B^{3}\left(\frac{r+m}{r-m}\right)^{\frac{3}{4 A}+\frac{A}{4}} \exp \left(3 C\left(\frac{r+m}{r-m}\right)^{A}\right)} \\
-8 a A^{2} C B\left(\frac{r+m}{r-m}\right)^{\frac{1}{4 A}+\frac{3 A}{4}} \cdot \exp \left(C\left(\frac{r+m}{r-m}\right)^{A}\right) d t^{2}, \\
\Phi=4 \sqrt{a} A C\left(\frac{r+m}{r-m}\right)^{A}, \quad x=B\left(\frac{r+m}{r-m}\right)^{\frac{1}{4 A}-\frac{A}{4}} \cdot \exp \left(C\left(\frac{r+m}{r-m}\right)^{A}\right)
\end{gathered}
$$

mit

$$
a>0, \quad A \neq 0, \quad C>0, \quad B>0, \quad m \neq 0, \quad|m|<r<\infty .
$$

Auch diese Metrik ist für $r \rightarrow \infty$ asymptotisch Minkowskisch und für $r \rightarrow 0$ singulär. Das singuläre Verhalten ist qualitativ so wie im vorangehenden Fall.

2. $a<0$. Hierbei ist

$$
\begin{gathered}
Q=\frac{d r^{2}+\left(r^{2}+m^{2}\right)\left(d \mathfrak{g}^{2}+\sin ^{2} \vartheta d \varphi^{2}\right)}{-2 a A^{2} C B^{3} \exp \left(\left(\frac{A}{4}-\frac{3}{A}\right) \operatorname{arctg} \frac{r}{m}+3 C \exp \left(A \operatorname{arctg} \frac{r}{m}\right)\right)} \\
-(-2 a) A^{2} C B \exp \left(\left(\frac{3 A}{4}-\frac{1}{A}\right) \operatorname{arctg} \frac{r}{m}+C \exp \left(A \operatorname{arctg} \frac{r}{m}\right)\right) d t^{2}, \\
\Phi=2 \sqrt{-a} A C \exp \left(A \operatorname{arctg} \frac{r}{m}\right), \\
\varkappa=B \exp \left(-\left(\frac{1}{A}+\frac{A}{4}\right) \operatorname{arctg} \frac{r}{m}+C \exp \left(A \operatorname{arctg} \frac{r}{m}\right)\right)
\end{gathered}
$$

mit

$$
a<0, \quad A \neq 0, \quad C>0, \quad B>0, \quad m \neq 0, \quad-\infty<r<\infty .
$$

Diese Lösung ist qualitativ so beschaffen wie die unter 1 . besprochene; sie ist zweiseitig asymptotisch Mrnkowskrsch und singularitätenfrei. 